\title{
Mutations of Genes Involved in the Innate Immune System as Predictors of Sepsis in Very Low Birth Weight Infants
}

\author{
PETER AHRENS, EVELYN KATTNER, BIRGIT KÖHLER, CHRISTOPH HÄRTEL, \\ JÜRGEN SEIDENBERG, HUGO SEGERER, JENS MÖLLER, AND WOLFGANG GÖPEL FOR THE \\ GENETIC FACTORS IN NEONATOLOGY STUDY GROUP \\ Department of Pediatrics, University of Lübeck, 23538 Lübeck [P.A., C.H., W.G.]; Childrens Hospital auf \\ der Bult, 30173 Hannover [E.K.]; Department of Pediatrics University of Marburg, 35037 Marburg \\ [B.K.]; Elisabeth Kinderkrankenhaus, 26133 Oldenburg [J.S.]; Childrens Hospital St. Hedwig, 93049 \\ Regensburg [H.S.]; and Childrens Hospital Saarbrücken, 66026 Saarbrücken [J.M.], Germany
}

\begin{abstract}
Mutations of genes involved in the innate immune system have been reported to be associated with an increased sepsis rate in adults. We determined the $-159 \mathrm{~T}$ mutation of the CD14 gene, the $896 \mathrm{G}$ mutation of the toll-like receptor 4 gene, the $3020 \mathrm{insC}$ mutation of the NOD2 gene (NOD2-3020insC), the IL-6 174G/C promoter polymorphism $(I L 6-174 \mathrm{G} / \mathrm{C})$, and the mannosebinding lectin genotype and their association to the subsequent development of neonatal sepsis in a large cohort of very low birth weight (VLBW) infants. Fifty (14\%) of 356 VLBW infants developed blood culture-proven sepsis during their stay in the hospital. VLBW infants carrying the NOD2-3020insC allele ( $n$ $=15)$ and the IL6-174G allele $(n=121)$ had a significantly higher rate of blood culture-proven sepsis (33\% and $19.8 \%$, respectively) than VLBW infants without these genotypes ( $p=$ 0.046 and 0.035 , respectively). In a multivariate logistic regression analysis, gestational age less than $28 \mathrm{wk}$ (odds ratio, 3.2; $95 \%$ confidence interval, $1.7-6.0 ; p<0.001$ ) and the homozygous IL6-174G allele (odds ratio, 1.9; 95\% confidence interval, $1.0-3.9 ; p=0.039$ ) were predictive for the development of
\end{abstract}

sepsis, whereas the NOD2-3020insC allele was only of borderline significance (odds ratio, 3.2; 95\% confidence interval, 1.0 $10.4 ; p=0.052$ ). VLBW infants with repeated episodes of sepsis had higher frequencies of the NOD2-3020insC and IL6-174G allele. The increased sepsis rate of homozygous IL6-174G carriers was especially related to an increase in Gram-positive infections, and was not observed in VLBW infants who received prophylaxis with teicoplanin (frequency of Gram-positive sepsis in homozygous IL6-174G carriers without prophylaxis $16.5 \%$ versus $2.4 \%$ in homozygous $I L 6-174 \mathrm{G}$ carriers with prophylaxis; $p=0.033)$. (Pediatr Res 55: 652-656, 2004)

LPS, lipopolysaccharide

Abbreviations

MBL, mannose-binding lectin

NF- $\mathbf{B} \mathbf{B}$, nuclear-factor kappa B

TLR, toll-like receptor

VLBW, very low birth weight
Although advances in neonatal intensive care have led to improved survival, sepsis continues to be an important cause of death among VLBW infants $(1,2)$. Recent advances in our understanding of the innate immune system triggered the identification of specific point mutations that are associated with an altered response of the innate immune system.

CD14 is the main receptor for bacterial LPS and is expressed on the surface of phagocytes. In adults, the homozygotic $-159 \mathrm{~T}$ mutation of the CD14 gene (CD14-159T) is associated

Received February 4, 2003; accepted September 26, 2003.

Correspondence: Peter Ahrens, M.D., Klinik für Kinder- und Jugendmedizin, Universitätsklinikum Schleswig-Holstein, Ratzeburger Allee 160, 23538 Lübeck, Germany; e-mail: ahrens@paedia.ukl.mu-luebeck.de

Supported by the Deutsche Forschungsgemeinschaft, grant-no. Go 955/1-1.

DOI: 10.1203/01.PDR.0000112100.61253.85 with a high mortality rate in sepsis (3). The TLR4 is a coreceptor for LPS, harboring a transmembrane domain, which is important for intracellular signaling. The $896 \mathrm{G}$ mutation of the TLR4 (TLR4-896G) leads in vitro to a reduced NF- $\kappa \mathrm{B}$ activation after LPS stimulation, and in vivo to a reduced systemic inflammatory response after LPS inhalation (4). This mutation was found frequently in adults who developed septic shock (5), suggesting an association of this mutation with sepsis. NOD2 is a gene that also confers responsiveness to bacterial LPS. The NOD2-3020insC mutation is associated in vitro with reduced $\mathrm{NF}-\kappa \mathrm{B}$ response after stimulation with several Gram-negative bacteria (6) and is associated with Crohns' disease (6-8). IL-6 is an important proinflammatory cytokine. The homozygous $-174 \mathrm{G}$ allele of the IL6-174G/C promoter polymorphism is associated with reduced IL-6 levels 
in healthy newborns (9). MBL is participating in the innate immune defense by opsonizing various microorganisms for phagocytosis. Heterozygous mutations of the MBL gene ( $M B L$ $\mathrm{B} / \mathrm{C} / \mathrm{D})$ are associated with reduced MBL serum levels and an increased sepsis rate in immunosuppressed patients (10).

Because a reduced response of the innate immune system in carriers of these mutations might be associated with a higher rate of bacterial infections in VLBW infants, the purpose of our study was to evaluate the usefulness of these genetic markers in predicting blood culture-proven sepsis in VLBW infants.

\section{METHODS}

Between December 1999 and November 2002, 535 VLBW infants were enrolled in an open multicenter study concerning the influence of genetic factors on the clinical course of VLBW infants. In November 2002 follow-up was completed in 372 VLBW infants. Because polymorphism frequencies differ widely among populations, we decided to exclude infants of African and Far Eastern descent $(n=16)$. The remaining 356 VLBW infants formed our study population.

Follow-up of prospectively enrolled VLBW infants was complete before discharge from the hospital. All parents had given informed written consent. Sepsis in VLBW infants was defined as blood culture-proven sepsis (clinical signs of sepsis and positive blood culture) at any time during the stay in the hospital. The clinical data of all patients were documented and coded before mutation analysis.

DNA samples of the infants were extracted from buccal swabs. DNA was extracted with a commercially available kit (Qiagen, Hilden, Germany). All polymorphisms were detected by PCR and restriction enzyme digestion. Primer and DNA sequences were selected from previously published reports $(4$, 7, 11-13). Primer pairs for detection of the CD14-159T mutation were 5'-GTG CCA ACA GAT GAG GTT CAC-3' and 5'-GCC TCT GAC AGT TTA TGT AAT C-3' (AvaII digest); for the TLR4-896G mutation, 5'ATA CTT AGA CTA CTA CCT CCA-3' and 5'CTT TGT TGG AAG TGA AAG TAA GCC-3' (NcoI digest); for the NOD2-3020insC -mutation, 5'CTG AGC CTT TGT TGA TGA GC-3' and 5'-TCT TCA ACC ACA TCC CCA TT-3' (NlaIV digest); for the IL6-174G/C polymorphism, 5'TGA CTT CGA CTT TAC TCT TGT-3' and 5'-CTG ATT GGA AAC CTT ATT AAG-3' (NlaIII digest); and for the $M B L$ genotype, 5'-AGT CGA CCC AGA TTG TAG GAC AGAG-3' and 5'-AGG ATC CAG GCA GTT TCC TCT GGA AGG-3' (BamI and MboII digest), and 5'-CAT CAA
CGG CTT CCC AGG CAA AGA CGC G-5' and 5'-AGG ATC CAG GCA GTT TCC TCT GGA AGG-3' (MluI digest).

Our power calculation was based on the assumption that the expected frequency of sepsis in VLBW infants is $20 \%$ and that the carrier status of a specific mutation is associated with an increased risk of $50 \%$. The expected carrier frequencies for the homozygous $C D 14-159 \mathrm{~T}$ mutation, the heterozygous or homozygous TLR4-896G and NOD2-3020insC mutations, homozygous -174G-IL6 promoter polymorphism, and the $M B L$ B/C/D genotype were $20 \%$ (14), 12\% (4), $8 \%$ (8), 37\% (12), and $40 \%(15)$, respectively. On the basis of these assumptions, a number of 230 infants would be sufficient $(\alpha, 0.05$; power, 0.8 ; two-sided test) to test our hypothesis.

All parts of the study were approved by the local committee on research in human subjects of the Medical University of Lübeck. Hypotheses were evaluated with Fisher exact test, Mann-Whitney $U$ test, and multivariate logistic regression models.

\section{RESULTS}

Ten participating centers included 356 patients (median, 21; range, 1-136). The clinical data and the frequencies of homozygous CD14-159T allele, heterozygous or homozygous TLR4-896G allele, heterozygous or homozygous NOD23020 insC allele, homozygous IL6-174G allele, and heterozygous or homozygous $M B L \mathrm{~B} / \mathrm{C} / \mathrm{D}$ genotype in VLBW infants are given in Table 1. We observed no statistical differences in clinical data between infants with and without specific mutations.

The frequency of blood culture-proven sepsis was $14 \%$ in the whole group $(n=356)$, ranging from $0 \%$ (none of 28 infants) to $100 \%$ ( 1 of 1 infant) among different centers. The frequency of blood culture-proven sepsis in two centers that included the majority of patients (110 and 136 infants) was 19.1 and $7.4 \%$, respectively.

The frequencies of blood culture-proven sepsis in VLBW infants with and without mutations of the innate immune system are given in Figure 1. VLBW infants who did not carry any of the mutations $(n=119)$ had a lower frequency of blood culture-proven sepsis than infants who carried any of the mutations tested ( $n=237)$; however, this difference was not significant ( 11.8 versus $15.2 \%, p=0.42$, Fisher's exact test). In VLBW infants carrying mutations of the innate immune system, the amount of increase in sepsis rate was quite different, reaching statistical significance for carriers of the homozy-

Table 1. Clinical data of VLBW infants with and without mutations of the innate immune system

\begin{tabular}{|c|c|c|c|c|c|c|c|}
\hline & $\begin{array}{c}\text { All } \\
(n=356)\end{array}$ & $\begin{array}{l}\text { No mutation } \\
(n=105)\end{array}$ & $\begin{array}{l}C D 14-159 \mathrm{~T} \\
(n=72)\end{array}$ & $\begin{array}{l}\text { TLR4-896G } \\
(n=34)\end{array}$ & $\begin{array}{l}\text { NOD2-3020insC } \\
\quad(n=15)\end{array}$ & $\begin{array}{l}\text { IL6-174G } \\
(n=121)\end{array}$ & $\begin{array}{c}M B L \mathrm{~B} / \mathrm{C} / \mathrm{D} \\
(n=116)\end{array}$ \\
\hline Birth weight (g) & $1073(295)$ & $1057(296)$ & $1041(296)$ & $1111(311)$ & $1048(337)$ & 1101 (284) & $1105(298)$ \\
\hline $\mathrm{GA}<28 \mathrm{wk}$ & 38 & 40 & 40 & 35 & 40 & 40 & 32 \\
\hline Multiple birth & 36 & 39 & 35 & 38 & 47 & 40 & 30 \\
\hline Antenatal steroids & 64 & 67 & 67 & 65 & 73 & 67 & 58 \\
\hline Frequency of mutation & Ref. & 29.5 & 20 & 9.6 & 4.2 & 34 & 33 \\
\hline
\end{tabular}

Birth weight is given as mean (SD); all other data are given as percentages. There were no significant differences between infants with and without mutations of the innate immune system.

Abbreviation used: GA, gestational age. 


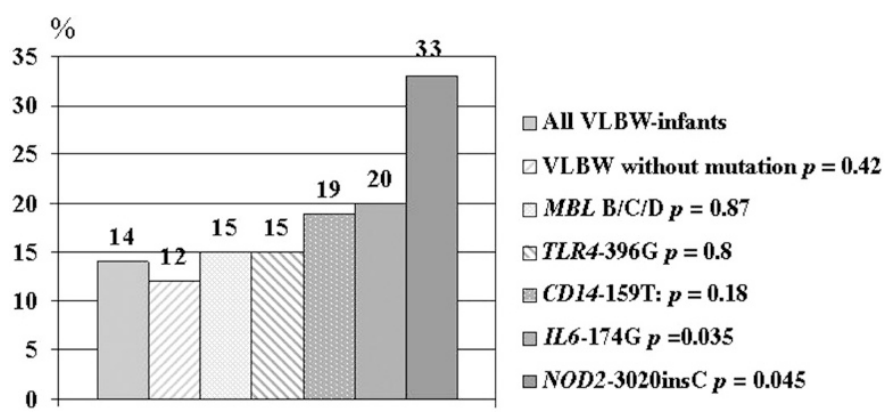

Figure 1. Frequency of blood culture-proven sepsis in 356 VLBW infants with or without mutations of the innate immune system. Probability values are given for frequencies of blood culture-proven sepsis in infants with a specific mutation $v s$ infants without the mutation (Fisher's exact test, two-sided).

gous IL6-174G mutation $(n=121$, sepsis rate $19.8 \%$ versus $11.1 \%$ in noncarriers of the mutation, $p=0.035$, Fisher's exact test) and carriers of the NOD2-3020insC mutation ( $n=15$, sepsis rate $33 \%$ versus $13.2 \%$ in noncarriers of the mutation, $p=0.045$, Fisher's exact test).

To rule out possible confounding, we performed a multivariate logistic regression analysis including gestational age, sex, multiple birth, antenatal treatment with glucocorticoids, and all mutations studied as independent variables and blood culture-proven sepsis as the dependent variable (Table 2). After stepwise exclusion of nonsignificant independent variables, only gestational age less than 28 wk (odds ratio, 3.2; 95\% confidence interval, 1.7-6.0; $p<0.001)$ and homozygous carrier status of the IL6-174G mutation (odds ratio, 1.9; 95\% confidence interval, 1.0-3.6; $p=0.039$ ) were significant predictors of blood culture-proven sepsis, whereas the carrier status of the NOD2-3020insC mutation was of only borderline significance (odds ratio, 3.2; 95\% confidence interval, $1-10.4 ; p=0.052$ ).

Furthermore, we analyzed the frequency of blood cultureproven sepsis in infants with and without the IL6-174G or NOD2-3020insC mutation; 306 of 356 infants had no sepsis, 42 infants one sepsis, seven infants two episodes of sepsis, and one infant suffered three events of blood culture-proven sepsis. The frequency of the IL6-174G or NOD2-3020insC mutation in these four groups was $34 \%, 48 \%, 57 \%$, and $100 \%$, demonstrating a statistically significant trend toward higher rates of repeated episodes of sepsis in infants carrying the IL6-174G or NOD2-3020insC mutation ( $p=0.026$, Mann-Whitney $U$ test).

The type of infection in VLBW infants carrying the IL6$174 \mathrm{G}$ or the NOD2-3020insC mutation is given in Table 3 . The increased sepsis rate in carriers of the homozygous IL6-174G mutation is related to an increase of sepsis caused by Grampositive bacteria. Two of the participating centers instituted
Table 3. Type of infection in infants with specific mutations of the innate immune system

\begin{tabular}{lccc}
\hline & $\begin{array}{c}\text { Gram-positive } \\
\text { infections }\end{array}$ & $\begin{array}{c}\text { Gram-negative } \\
\text { infections }\end{array}$ & $\begin{array}{c}\text { Other } \\
\text { infections }\end{array}$ \\
\hline All VLBW $(n=356)$ & 6.7 & 2.5 & 5.3 \\
IL6-174G $(n=121)$ & $\mathbf{1 1 . 6}$ & 3.3 & 5.8 \\
NOD2-3020insC $(n=15)$ & 13.3 & 0.0 & $\mathbf{2 0}$ \\
\hline
\end{tabular}

All data are given as percentages of all infants or percentages of infants with specific mutations. Gram-positive infections include infections with Staphylococcus aureus $(n=11)$, Staphylococcus epidermidis $(n=12)$ and Streptococci $(n=6)$; Gram-negative infections include infections with Escherichia coli $(n=5)$, Klebsiella $(n=1)$, Enterobacter $(n=2)$, Serratia $(n=0)$, Proteus $(n=0)$, and Pseudomonas sp. $(n=1)$. Other infections include Listeria $(n=$ $0)$, anaerobic infections $(n=0)$, fungi $(n=4)$, and other Gram-negative or Gram-positive infections not listed above $(n=17)$. Significant differences between carriers and noncarriers of the mutation are given in bold $(p<0.05$, Fisher's exact test, two-sided).

routine prophylaxis against Gram-positive infections (16), consisting of daily administration of $6 \mathrm{mg}$ teicoplanin/ $\mathrm{kg}$ body weight per day if an i.v. catheter is necessary in all infants with a birth weight below $1500 \mathrm{~g}$ (center $4, n=136$ infants) or infants with a birth weight below $1000 \mathrm{~g}$ (center $10, n=1$ infant). Therefore, we evaluated the rate of different types of infections in VLBW infants with and without teicoplanin prophylaxis stratified to their IL6-174G genotype. Because there were large differences among the rates of infection in different centers, we furthermore present data of the two largest centers, numbers 4 (routine teicoplanin prophylaxis for all VLBW infants) and 6 (no prophylaxis; Table 4). The higher rate of blood culture-proven sepsis in carriers of the homozygous IL6-174G allele was related to the high rate of Grampositive infections in VLBW infants who did not received teicoplanin prophylaxis. Furthermore, homozygous carriers of the IL6-174C genotype had a relatively lower risk of Gram-positive sepsis than carriers of the heterozygous IL6$174 \mathrm{G} / \mathrm{C}$ genotype. The trend toward higher rates of Grampositive infections in homozygous carriers of the IL6-174G allele was detectable, even when only one large center was analyzed. No differences between carriers of different IL6174 alleles were observed with regard to Gram-negative infections and in VLBW infants who were treated with teicoplanin prophylaxis (Table 4).

\section{DISCUSSION}

Recent advances in understanding the molecular basis of the innate immune system led to the identification of certain point mutations of genes involved in the early recognition of bacteria and associated with a reduced host response $(3-6,9,10)$. The

Table 2. Multivariate logistic regression analysis: gestational age and homozygous IL6-174G mutation as independent predictors of blood culture-proven sepsis

\begin{tabular}{|c|c|c|c|c|c|}
\hline Independent variable & $\begin{array}{c}\text { VLBW with } \\
\text { sepsis }(n=50)\end{array}$ & $\begin{array}{l}\text { VLBW without } \\
\text { sepsis }(n=306)\end{array}$ & OR & $95 \% \mathrm{CI}$ & $p$ value \\
\hline Gestational age $<28$ wk & 62 & 34 & 3.2 & $1.7-6.0$ & $<0.001$ \\
\hline Heterozygous NOD2-3020insC & 10 & 3.3 & 3.2 & $1.0-10.4$ & 0.052 \\
\hline Homozygous IL6-174G & 48 & 32 & 1.9 & $1-3.6$ & 0.039 \\
\hline
\end{tabular}

Frequencies of independent variables in VLBW infants with and without blood culture-proven sepsis are given as percent values.

Abbreviations used: OR, odds ratio; CI, confidence interval. 
Table 4. Frequency of blood culture-proven sepsis in VLBW infants with different IL6-174 genotypes stratified to type of infection and teicoplanin prophylaxis

\begin{tabular}{|c|c|c|c|c|c|}
\hline \multirow[b]{2}{*}{ Type of infection, subgroup } & \multicolumn{5}{|c|}{ Frequency of blood culture-proven sepsis in infants according to IL6-174 genotype } \\
\hline & All infants & IL6-174GG & IL6-174G/C & IL6-174CC & $p$ value* \\
\hline All kind of infections, all infants & $50 / 356(14 \%)$ & $24 / 121(19.8 \%)$ & $21 / 198(10.6 \%)$ & $5 / 37(13.5 \%)$ & 0.035 \\
\hline Gram-negative and other, all infants & $26 / 356(7.3 \%)$ & $11 / 121(9.1 \%)$ & $14 / 198(7.1 \%)$ & $3 / 37(8.1 \%)$ & 0.54 \\
\hline Gram-positive, all infants & $24 / 356(6.7 \%)$ & $14 / 121(11.6 \%)$ & $8 / 198(4 \%)$ & $2 / 37(5.4 \%)$ & 0.013 \\
\hline Gram-positive, all infants with teicoplanin prophylaxis & $3 / 137(2.2 \%)$ & $1 / 42(2.4 \%)$ & $1 / 82(1.2 \%)$ & $1 / 13(7.7 \%)$ & 1.0 \\
\hline Gram-positive, all infants without teicoplanin prophylaxis & $21 / 219(9.6 \%)$ & $13 / 66(16.5 \%)$ & $7 / 116(6 \%)$ & $1 / 24(4.2 \%)$ & 0.015 \\
\hline Gram-positive, study center 4 , teicoplanin prophylaxis & $3 / 136(2.2 \%)$ & $1 / 42(2.4 \%)$ & $1 / 81(1.2 \%)$ & $1 / 13(7.7 \%)$ & 1.0 \\
\hline Gram-positive, study center 6 , no teicoplanin prophylaxis & $11 / 110(10 \%)$ & $8 / 37(21.6 \%)$ & $3 / 52(5.8 \%)$ & $0 / 21(0 \%)$ & 0.006 \\
\hline
\end{tabular}

* $p$ values are given for infants carrying the $-174 \mathrm{GG}$ genotype $v s$ infants carrying the $-174 \mathrm{G} / \mathrm{C}$ or $-174 \mathrm{C} / \mathrm{C}$ genotype (Fisher's exact test, two-sided).

scope of our study was the association of these mutations with blood culture-proven sepsis in a large cohort of VLBW infants. Although the sepsis rate was higher in infants carrying any of the mutations studied, these differences were statistically significant only for the NOD2-3020C mutation and the IL6-174G mutation.

The NOD2-3020insC mutation was first described in 2001 as a risk factor for Crohn's disease. NOD2 is expressed in monocytes and carries a binding domain for bacterial LPS (17). The 3020insC mutation resulted in a frameshift at the second nucleotide of codon 1007 followed by a premature stop codon. The truncated NOD2 protein contained 1007 amino acids instead of 1040 amino acids of the wild-type NOD2 protein. In vitro studies using HEK293T cells transfected with wild-type or mutant NOD2 receptor demonstrated that LPS from various bacteria induced NF- $\kappa \mathrm{B}$ activation in wild-type but not in cells transfected with NOD2-3020insC (6). The frequency of the heterozygous NOD2-3020insC mutation in our cohort of preterm infants was $4.2 \%$, which is in line with previous reports $(6-8)$. At this time there are no studies concerning the association of the NOD2-3020insC mutation and septic events in patients of any age.

The $I L 6-174 \mathrm{G} / \mathrm{C}$ polymorphism and its association with septic events in adults was studied recently. Allele frequencies did not differ significantly between patients with or without sepsis, and median systemic IL-6 levels were not associated with the genotype. However, in patients who finally died because of sepsis, significantly fewer carriers of the homozygous IL6-174G genotype were observed (18). Studies concerning the effect of different IL6-174 genotypes on IL-6 plasma and C-reactive protein levels gave conflicting results. Some groups report no or only moderate effects $(19,20)$, whereas others report lower IL-6 plasma levels in healthy carriers of the IL6-174C allele (12) and - in contrast to these data- higher baseline C-reactive protein levels in carriers of the $\mathrm{C}$ allele (21). The only study concerning the genotype-dependent IL-6 production in healthy newborns reported significantly higher IL-6 plasma levels in carriers of the IL6-174C allele compared with infants homozygous for the IL6-174G allele (9). We therefore decided to evaluate the hypothesis that VLBW infants carrying the homozygous $I L 6-174 \mathrm{G}$ genotype are at an increased risk for the development of sepsis.

We observed a significant association between the heterozygous NOD2-3020insC and the homozygous IL6-174G genotype and the development of blood culture-proven sepsis in
VLBW infants. The latter association was not influenced by other known or possible risk factors for the development of sepsis in VLBW infants. Furthermore, VLBW infants with repeated episodes of septicemia were more frequently carriers of the mutations than infants without or with only one blood culture-proven sepsis. The increased sepsis rate of homozygous carriers of the IL6-174G mutation was especially related to an increase of Gram-positive infections. Infants receiving prophylaxis with teicoplanin had a low sepsis rate even if they were carriers of the homozygous IL6-174G mutation, whereas noncarriers of the mutation had a low sepsis rate even if they had not had any prophylactic treatment. The number of infants needing to be treated with teicoplanin prophylaxis to prevent one blood culture-proven Gram-positive sepsis calculated on the basis of our data would be seven infants in carriers of the mutation, but 28 infants in noncarriers of the mutation.

\section{CONCLUSIONS}

Our data suggest that teicoplanin prophylaxis stratified to the individual genetic risk of a given VLBW infant to develop Gram-positive sepsis might be useful in reducing the number of unnecessary prophylactic antibiotic treatments. However, it must kept in mind that this association study is not proof of a causal relationship between the IL6-174G allele and Grampositive infections, but might be related to linkage to other polymorphisms. Furthermore, inasmuch as frequencies of blood culture-proven sepsis varied widely among different centers of our study, a possible association between IL6-174G and susceptibility to Gram-positive sepsis must be evaluated by randomized controlled trials, because the aim of our study was the association between specific mutations of the innate immune system and the development of sepsis in VLBW infants, and the differences observed between VLBW infants with and without prophylactic antibiotic treatment might be the result of other confounding factors.

Acknowledgments. The authors thank Anja Sewe, Lynn Ellenberg and Sabine Ziesenitz for excellent laboratory assistance, Birgit Roenspiess for skillful data collection, all doctors and nurses of the participating hospitals, and especially all infants and their parents for their support. 


\section{APPENDIX}

Other investigators of the Genetic Factors in Neonatology Study Group: K. Albrecht (Bremen); R. Jensen (Heide); A. Schwabe (Oldenburg); A. Leonhardt, M. Raab (Marburg); A. Warnecke (Osnabrück); and A. v.d.Wense (Hamburg).

\section{REFERENCES}

1. Stoll BJ, Hansen N, Fanaroff AA, Wright LL, Carlo WA, Ehrenkranz RA, Lemons JA, Donovan EF, Stark AR, Tyson JE, Oh W, Bauer CR, Korones SB, Shankaran S, Laptook AR, Stevenson DK, Papile LA, Poole WK 2002 Late-onset sepsis in very low birth weight neonates: the experience of the NICHD Neonatal Research Network. Pediatrics 110:285-291

2. Stoll BJ, Hansen N, Fanaroff AA, Wright LL, Carlo WA, Ehrenkranz RA, Lemons JA, Donovan EF, Stark AR, Tyson JE, Oh W, Bauer CE, Korones SB, Shankaran S, Laptook AR, Stevenson DK, Papile LA, Poole K 2002 Changes in pathogens causing early-onset-sepsis in very-low-birth-weight infants. N Engl J Med 347:240-247

3. Gibot S, Cariou A, Drouet L, Rossignol M, Ripoll L 2002 Association between a genomic polymorphism within the CD14 locus and septic shock susceptibility and mortality rate. Crit Care Med 30:969-973

4. Arbour NC, Lorenz E, Schutte C, Zabner J, Kline JN, Jones M, Frees K, Watt JL, Schwartz DA 2000 TLR4 mutations are associated with endotoxin hyporesponsiveness in humans Nat Genet 25:187-191

5. Lorenz E, Mira JP, Frees KL, Schwartz DA 2002 Relevance of mutations in the TLR4 receptor in patients with Gram-negative septic shock. Arch Intern Med 162:1028 1032

6. Ogura Y, Bonen DK, Inohara N, Nicolae DL, Chen FF, Ramos R, Britton H, Moran T, Karaliuskas R, Duerr RH, Achkar JP, Brant SR, Bayless TM, Kirschner BS Hanauer SB, Nunez G, Cho JH 2001 A frameshift mutation in NOD2 associated with susceptibility to Crohn's disease. Nature 411:603-606

7. Hugot J-P, Chamaillard M, Zouali H, Lesage S, Cézard J-P, Belaiche J, Almer S, Tysk C, O'Morain CA, Gassull M, Binder V, Finkel Y, Cortot A, Modigliani R, Laurent-Puig P, Gower-Rousseau C, Macry J, Colombel JF, Shabatou M, Thomas G 2001 Association of NOD2 leucine-rich repeat variants with susceptibility to Crohn's disease. Nature 411:599-603

8. Hampe J, Cuthbert A, Croucher PJP, Mirza MM, Mascheretti S, Fisher S, Frenzel H, King K, Hasselmeyer A, MacPherson AJS, Bridger S, Van Deventer S, Forbes A, Nikolaus S, Lennard-Jones JE, Foelsch UR, Krawczak M, Lewis C, Schreiber S, Mathew CG 2001 Association between insertion mutation in NOD2 gene and Crohn's disease in German and British populations. Lancet 357:1925-1928
9. Kilpinen S, Hulkkonen J, Wang XY, Hurme M 2001 The promoter polymorphism of the interleukin- 6 gene regulates interleukin- 6 production in neonates but not in adults. Eur Cytokine Netw 12:62-68

10. Mullinghan CG, Heatley S, Doherty K, Szabo F, Grigg A, Hughes TP, Schwarer AP, Szer J, Tait BD, To LB, Bardy PG 2002 Mannose-binding lectin gene polymorphisms are associated with major infection following allogeneic hemopoietic stem cell transplantation. Blood 99:3524-3529

11. Baldini M, Lohman IC, Halonen M, Erickson RP, Holt PG, Martinez FD 1999 A polymorphism in the $5^{\prime}$ flanking region of the CD14 gene is associated with circulating soluble CD14 levels and with total serum immunoglobulin E. Am J Respir Cell Mol Biol 20:976-983

12. Fishman D, Faulds G, Jeffery R, Mohammed-Ali V, Yudkin JS, Humphries S, Woo P 1998 The effect of novel polymorphisms in the interleukin-6 (IL-6) gene on IL-6 transcription and plasma IL-6 levels, and an association with systemic-onset juvenile chronic arthritis. J Clin Invest 102:1369-1376

13. Madsen HO, Garred P, Thiel S, Kurtzhals JAL, Lamm LU, Ryder LP, Svejgaard A 1995 Interplay between promoter and structural gene variants control basal serum level of mannan-binding protein. J Immunol 155:3013-3020

14. Unkelbach K, Gardemann A, Kostrzewa M, Philipp M, Tillmanns H, Haberbosch W 1999 A new promoter polymorphism in the gene of lipopolysaccharide receptor CD14 is associated with expired myocardial infarction in patients with low atherosclerotic risk profile. Arterioscler Thromb Vasc Biol 19:932-938

15. Roy S, Knox K, Segal S, Griffiths D, Moore CE, Welsh KI, Smarason A, Day NP, McPheat WL, Crook DW, Hill AVS, Oxford Pneumococcal Surveillance Group 2002 MBL genotype and risk of invasive pneumococcal disease: a case-control study. Lancet 359:1569-1573

16. Möller JC, Nelskamp I, Jensen R, Reiss I, Kohl M, Gatermann S, Iven H, Gortner L 1997 Comparison of vancomycin and teicoplanin for prophylaxis of sepsis with coagulase negative staphylococci in very low birth weight infants. J Perinat Med 25:361-367

17. Inohara N, Ogura Y, Chen FF, Muto A, Nuñez G 2001 Human Nod1 confers responsiveness to bacterial lipopolysaccharides. J Biol Chem 276:2551-2554

18. Schluter B, Raufhake C, Erren M, Schotte H, Kipp F, Rust S, Van SH, Assmann G, Berendes E 2002 Effect of the interleukin-6 promoter polymorphism $(-174 \mathrm{G} / \mathrm{C})$ on the incidence and outcome of sepsis. Crit Care Med 30:32-37

19. Margaglione M, Bossone A, Cappucci G, Colaizzo D, Grandone E, Di Minno G 2001 The effect of interleukin-6 C/G-174 polymorphism and circulating interleukin- 6 on fibrinogen plasma levels. Haematologica 86:199-204

20. Heesen M, Bloemeke B, Heussen N, Kunz D 2002 Can the interleukin-6 response to endotoxin be predicted? Studies of the influence of a promoter polymorphism of the interleukin- 6 gene, gender, the density of the endotoxin receptor CD14, and inflammatory cytokines. Crit Care Med 30:664-669

21. Vickers MA, Green FR, Terry C, Mayosi BM, Julier C, Lathrop M, Ratcliffe PJ, Watkins HC, Keavney B 2002 Genotype at a promoter polymorphism of the interleukin-6 gene is associated with baseline levels of plasma C-reactive protein. Cardiovasc Res 53:1029-1034 